\title{
Meine Welt und mein Raum?
}

Liebe Leserinnen, liebe Leser

Meine Welt bestimme ich! - natürlich mit diversen Einschränkungen wie soziale und familiale, berufliche und sonstige umweltbedingte Verpflichtungen. Diese nehme ich mehr oder weniger gerne in Kauf, solange ich dabei nicht mein Recht verwirke, meine Lebenswelt und meinen Lebensraum zu gestalten und nach meinem Willen zu formen. Und wenn die Einschränkungen überhandnehmen, möchte ich zumindest ein Mitspracherecht haben. In letzter Zeit schwant mir zwar, dass meine gelobte Unabhängigkeit begrenzter ist als ich annehme und daran trägt nicht nur die Pandemie Schuld. Es ist immer eine Koexistenz zweier Kräfte: mein Ich als Individuum und mein Du als meine Umwelt! Mitunter kracht es zwar zwischen uns, aber wir wissen beide, dass wir in jeder Lebenslage aufeinander angewiesen sind und keiner ohne den anderen wirklich lebensfähig ist.

Auch habe ich bislang fälschlicherweise angenommen, dass man bei Pflegebedürftigkeit das Recht auf Mitwirkung an der Gestaltung des eigenen Lebensraums zumindest teilweise verliert, gleich einem unabdingbaren Gesetz, und die Waage sich bedenklich zur Seite der Umwelt neigt als künftigen „Bestimmer“. Ich bin sehr froh, dass mich die Autorinnen und Autoren dieser Ausgabe der NOVAcura eines Besseren belehren.

Wir treten in unsere Lebenswelt beschützt und geborgen ein, umhüllt von der Sicherheit der Gebärmutter. Die wesentlichen Reifungsprozesse geschehen dann ungeschützt, ausserhalb des Körpers der Mutter. Welche Einflüsse treffen uns dann und welche Bedeutung haben sie für unser ganzes Leben? - Diana Staudacher legt die wissenschaftlichen Grundlagen zum Thema vor. Mit dem Begriff des gemeinsam gestalteten Sozialraums erweitert $A n$ drea Grünenfelder die Diskussionen um Lebensraum und Lebenswelt und erörtert die Rahmenbedingungen eines professionellen Unterstützungssystem für die eigenständige Lebensführung älterer Menschen in einer integrierten Versorgung.

Hier knüpfen gleich zwei weitere Beiträge an, beide zeigen Möglichkeiten auf, Schutz und Geborgenheit des eigenen Lebensraums zu erhalten: Martin Herzberg beschreibt im Leben auf dem Dorf die Chancen einer natürlich schützenden Umwelt. Julia Sonntag erforscht das Community

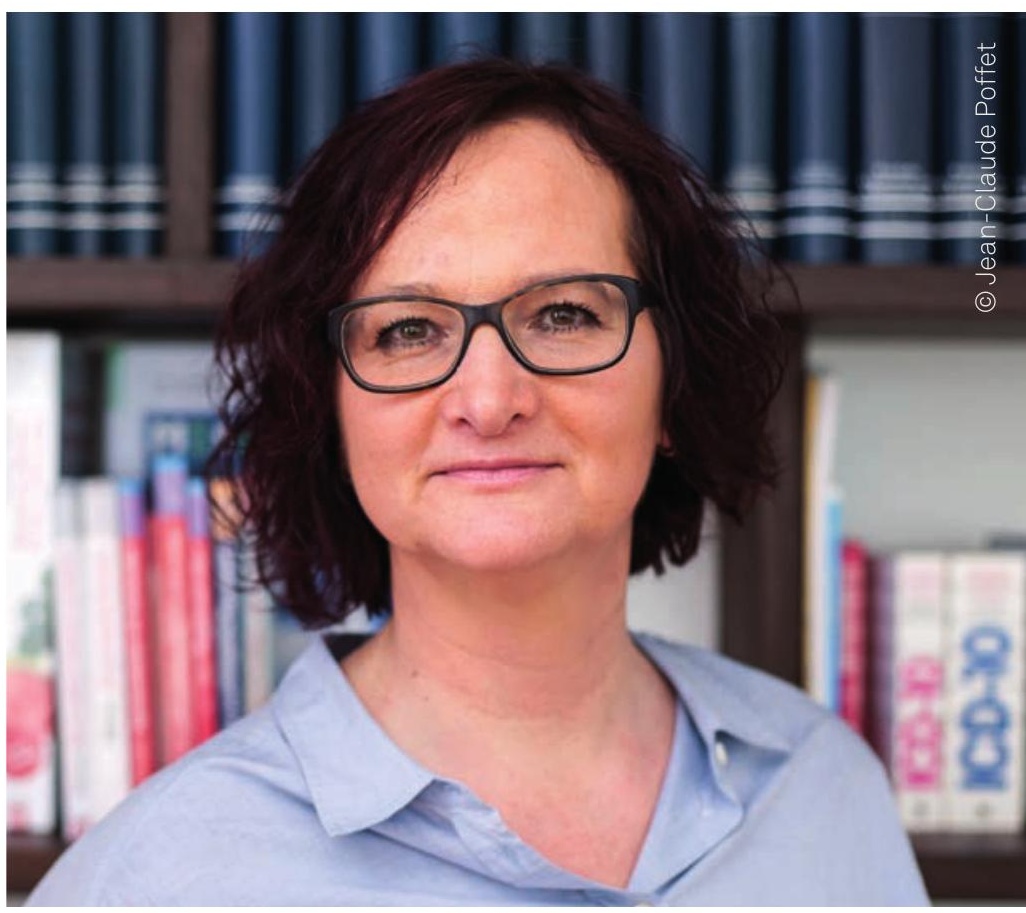

Health Nursing als Option professioneller Unterstützung im gewohnten Lebensraum bei Pflegebedürftigkeit.

Das Quartiersprojekt Nachbarschaft Bern unterstützt Menschen bei einschränkenden Bedingungen wie Krankheit und sozialer Isolation und gibt ihnen damit wieder Bedeutung und Wert zurück, was sie verloren glaubten: Binja Breitenmoser und Karen Torben-Nielsen lassen in ihrem Beitrag die älteren Menschen im Quartiersprojekt zu Wort kommen. Christine Sowinski vom Kuratorium Deutsche Altershilfe nennt im Interview Parameter für die Gestaltung eines Lebensraums, in dem sich die alten Menschen wohl fühlen können: Auch wenn eine Altenpflegeeinrichtung nicht das eigene Zuhause mehr ist, kann sie doch Wärme und Geborgenheit ausstrahlen und Wohlbefinden auslösen.

Den Lebensraum schützen und Pflegebedürftige beschützen: ein Ziel für unsere gemeinsame Zukunft? Mit diesen Gedanken wünsche ich Ihnen eine interessante Lektüre!

Mit herzlichen Grüssen,

Barbara Müller 


\section{Inhalt}

Editorial

Meine Welt und mein Raum?

Barbara Müller

Schwerpunkt

Der unverletzliche Raum

Diana Staudacher

Individuelles und gemeinschaftliches Wohnen im Alter

Andrea Grünenfelder

Wie nehmen über 80-jährige Menschen am sozialen Leben teil?

Binja Breitenmoser \& Karen Torben-Nielsen

Community Health Nursing

Julia Sonntag

Geranien, Gartenzaun, Community

Martin Herzberg

„Wie in einem schönen Landhotel““

Brigitte Teigeler

Medientipps zum Thema

Lena-Marie Wimmel

Fokus

„Für mein Kind ist das nichts."

Lotte Habermann-Horstmeier

Merkmale der geriatrischen Rehabilitation und pflegerische Aufgaben

Elke Steudter

\begin{tabular}{|c|c|c|}
\hline \multirow[t]{2}{*}{ Palliative Care } & $\begin{array}{l}\text { Kultursensible Pflege und Betreuung von Muslim_innen am Lebensende } \\
\text { Imane Henni Rached }\end{array}$ & 45 \\
\hline & $\begin{array}{l}\text { Palliative Care im südindischen Kerala } \\
\text { Sabine Millius }\end{array}$ & 49 \\
\hline Pflegen zu Hause & $\begin{array}{l}\text { Verbundenheit und Zufriedenheit mit der Wohnumgebung } \\
\text { bei Personen ab } 65 \text { Jahren } \\
\text { Alexander Seifert \& Klaus R. Schroeter }\end{array}$ & 53 \\
\hline \multirow[t]{2}{*}{ Bildung } & $\begin{array}{l}\text { Pflege und Forschung } \\
\text { Brigitte Teigeler }\end{array}$ & 56 \\
\hline & $\begin{array}{l}\text { Die Zukunftswerkstatt der Medizin } \\
\text { Stefan Müller }\end{array}$ & 59 \\
\hline \multirow[t]{2}{*}{ Dementia Care } & $\begin{array}{l}\text { Milieugestaltung in der Gerontopsychiatrie } \\
\text { Arben Duraku }\end{array}$ & 61 \\
\hline & $\begin{array}{l}\text { Menschen mit Demenz motivieren } \\
\text { Svenja Sachweh }\end{array}$ & 65 \\
\hline à propos & & 69 \\
\hline Vorschau/Termine & & 70 \\
\hline
\end{tabular}

